\title{
Effectiveness of Medtronic CareLink Express System in identifying patients with high-energy electrotherapy devices requiring clinically significant intervention
}

\author{
Marcin Grabowski ${ }^{1}$, Sabina Sadecka ${ }^{1}$, Andrzej Cacko ${ }^{1,2}$, \\ Marcin Michalak ${ }^{1}$, Leszek Blicharz ${ }^{1}$, Paweł Balsam ${ }^{1}$, Grzegorz Opolski ${ }^{1}$ \\ ${ }^{1} 1^{\text {st }}$ Department of Cardiology, Medical University of Warsaw, Poland \\ ${ }^{2}$ Department of Medical Informatics and Telemedicine, Medical University of Warsaw, Poland
}

\begin{abstract}
Background: The number of patients with implantable cardioverter-defibrillator (ICD) or cardiac resynchronization therapy and defibrillator (CRT-D) is growing. This results in high workloads for centers performing standard controls (SC) of these systems. Medtronic CareLink Express ${ }^{\circledR}$ (MCLE) is a solution enabling remote controls $(R C)$ of implantable devices. In cases of detecting arrhythmias or device malfunctioning, it automatically generates alarms transmitted to the controlling physician. The objective of the study was to evaluate the effectiveness of alarms generated by MCLE in identification of patients that require clinically significant intervention during $S C$.

Methods: A total of 119 patients (age $64 \pm 14,17$ women) implanted with ICD/CRT-D were included in the trial. Device reprogramming or pharmacological treatment modifications during SC were assessed as significant if they were required for optimal clinical management of the patient (effectiveness of antiarrhythmic and resynchronization therapy, also patient safety). Analysis of generated alarms was then performed to assess the effectiveness (sensitivity and specificity) of MCLE in identifying patients that require changes in therapy.

Results: Data from 129 transmissions and 129 subsequent SCs were analyzed. 179 alarms were recorded during 96 (74\%) transmissions. A total of 333 program changes of implanted devices took place during 107 (83\%) SCs. Device reprogramming was considered clinically significant in 27 cases Additionaly, 13 pharmacological treatment modifications were performed. Sensitivity and specificity of alarms generated by MCLE with regards to identification of patients requiring clinically significant intervention was $87 \%$ and $31 \%$, respectively. Finally, a statistically significant difference was observed in the number of clinically significant interventions among patients with and without MCLE alarms (33 [86.8\%] vs. 5 [13.2\%], $p=0.037$ ).

Conclusions: Medtronic CareLink Express ${ }^{\circledast}$ is an RC system enabling high-sensitivity supervision of patients with cardiac electrotherapy devices requiring changes of treatment during SC. (Cardiol J 2018; 25, 1: 81-86)
\end{abstract}

Key words: arrhythmic event, heart failure, high-energy electrotherapy devices, Medtronic CareLink Express, remote control

Address for correspondence: Marcin Grabowski, MD, PhD, $1^{\text {st }}$ Department of Cardiology, Medical University of Warsaw, ul. Banacha 1a, 02-097 Warszawa, Poland, tel: +48 226582 997, fax: +48 2259 91 957, e-mail: grabm@wum.edu.pl 


\section{Introduction}

Recent years brought significant advancements in therapy of patients suffering from heart failure and concomitant arrhythmias [1, 2]. Implantable cardioverter-defibrillators (ICD) and cardiac resynchronization therapy and defibrillators (CRT-D) are now universally implemented in long-term prevention of cardiac arrest $[3,4]$. An obvious downside associated with versatility of these highenergy electrotherapy devices is the necessity to control them regularly so that their malfunctioning or deterioration of patients' general state can be timely detected. Interventions performed during standard control (SC) include reprogramming of implanted device or adjustments of other clinical variables (e.g. pharmacological treatment) to optimize the therapy. These procedures increase workload in centers where SCs are performed. According to current standards, SC should be carried out at least once every 6-12 months in order to ensure appropriate clinical management of patients with a high-energy electrotherapy device [1]. Various remote monitoring systems that enable self-controls of implantable devices have been created with capabilities of reducing the burden related with $\mathrm{SCs}$. It is believed that remote controls (RC) hold the potential for decreasing frequency of SCs [5].

Medtronic CareLink Express ${ }^{\circledast}$ (MCLE) is a system available to patients managed by specialized centers performing SCs of implantable devices (Fig. 1). When MCLE detects arrhythmia or device malfunctioning during $\mathrm{RC}$, it automatically generates an alarm and transmits the data to the physician responsible for the follow-up of a given patient. Provided MCLE turns out to be a reliable remote monitoring system, it could limit the number of SCs and thus reduce the growing workload in control centers [6]. This could further encourage attempts to implement decision supporting systems and artificial intelligence in clinical practice to increase efficiency of ambulatory care.

The aim of the study was to evaluate the effectiveness of MCLE in identifying patients that require clinically significant intervention during SC.

\section{Methods}

Patients of a tertiary care center implanted with implantable cardioverter-defibrillator (ICD)/ /cardiac resynchronization therapy and defibrillator (CRT-D) were invited to take part in the trial. Refusal or inability to provide informed consent for personal and medical data collection in the MCLE

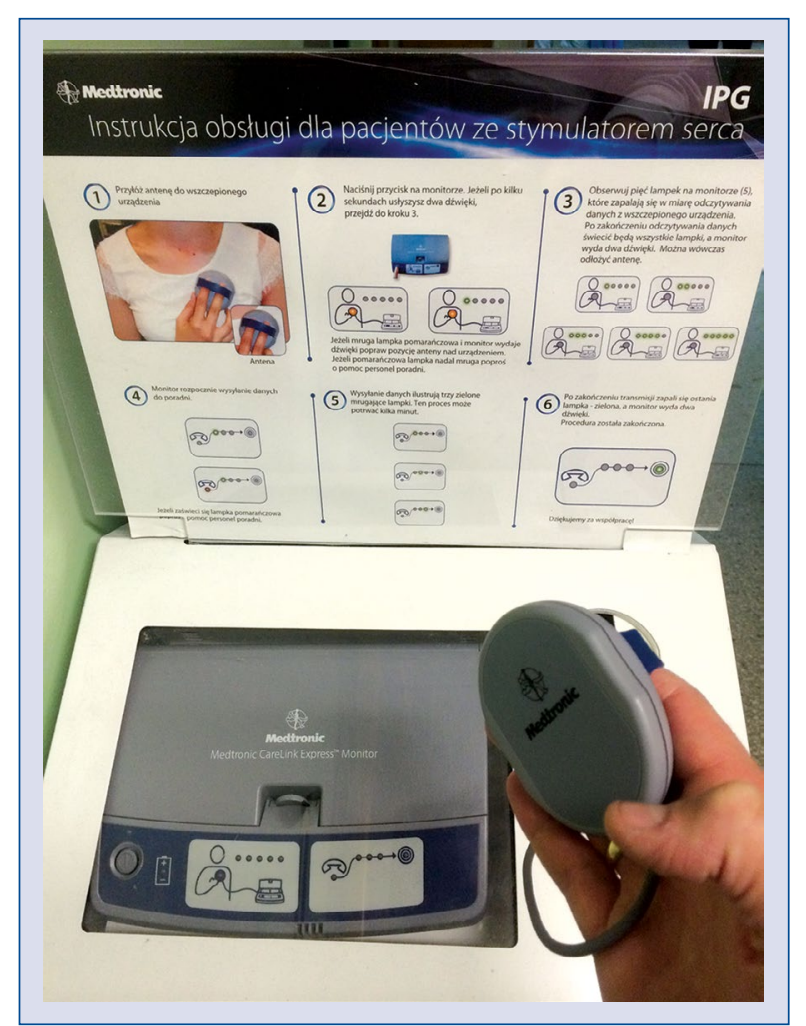

Figure 1. Medtronic CareLink Express system kit.

system were adopted as exclusion criteria. All subjects presented for an in-person evaluation of the implanted device between April and December 2013.

Every patient was instructed on how to use MCLE and performed his/her first interrogation before SC took place.

Medtronic CareLink Express ${ }^{\circledR}$ enables transmission of a wide range of information. The following data can be recorded during RCs performed with this system: current device programming, detected arrhythmic events, sensing and pacing parameters recorded in automatic tests, percentage of biventricular pacing (in case of CRT-D) and in some cases, assessment of heart failure compensation based on indirect criteria (mean physical activity, mean heart rate during the day and at night and transthoracic impedance). The data can be viewed in a Web browser immediately after transmission. Access to the data is secured with login and password. If a transmission contains information about the occurrence of arrhythmia or other anomalies detected by an implanted device, MCLE generates an alarm available to the user after logging in.

Medtronic CareLink Express ${ }^{\circledR}$ system is capable of generating automatic alarms reporting 
Table 1. Patients' characteristics

\begin{tabular}{lccc}
\hline Clinical factor & $\begin{array}{c}\text { Overall population } \\
\text { (n = 119 } \mathbf{~ t s )}\end{array}$ & $\begin{array}{c}\text { ICD subgroup } \\
\text { ( } \mathbf{n = 1 0 1} \text { ts) }\end{array}$ & $\begin{array}{c}\text { CRT-D subgroup } \\
\text { (n= 18 pts) }\end{array}$ \\
\hline Age [years] & $64 \pm 14$ & $63 \pm 14$ & $69 \pm 12$ \\
Males & $102(85.7 \%)$ & $86(85.1 \%)$ & $16(88.9 \%)$ \\
Time form device implantation [weeks] & $77 \pm 58$ & $79 \pm 52$ & $64 \pm 99$ \\
Primary prevention & $99(83.2 \%)$ & $82(81.2 \%)$ & $17(94.4 \%)$ \\
\hline
\end{tabular}

Data are shown as mean \pm standard deviation or number (percentage); pts - patients

the occurrence of sustained and non-sustained ventricular arrhythmias, supraventricular arrhythmias, applied low- and high-energy antiarrhythmic interventions, decreased percentage of resynchronizing stimulation, reduced transthoracic impedance, increased pacing threshold and incorrect lead impedance in a low or high-energy circuit.

Every patient presented for SC directly after a transmission. Data such as detected arrhythmic events, malfunction of the implanted system, ICD/CRT-D reprogramming and pharmacological treatment changes were recorded in the patient's history. Alarms generated by MCLE as well as all cases of device reprogramming or pharmacotherapy changes were analyzed. Changes made in device settings were divided into clinically significant and non-significant. Criteria of clinical significance were fulfilled if reprogramming (1) concerned ventricular arrhythmia detection zone or antiarrhythmic therapy, discriminators of supraventricular arrhythmias, pacing mode, basic pacing rate and atrioventricular delay, and (2) was required for the effectiveness of antiarrhythmic and resynchronization therapy as well as for patient safety. Two experts analyzed the fulfilment of reprogramming criteria during each SC.

Data from transmissions via MCLE were then compiled with observations from subsequent SCs. Sensitivity and specificity of alarms generated by MCLE were analyzed to assess clinical usefulness of the system (i.e. ability to identify patients requiring clinically significant ICD/ /CRT-D reprogramming or medication changes during $\mathrm{SC}$ ).

This was achieved by comparing the rate of clinically significant reprogramming in patients with and without MCLE alarms. Statistical analysis was performed using Statistica 12. Differences between groups were analyzed with the $\chi^{2}$ test. Statistically significant differences were defined with $\mathrm{p} \leq 0.05$.

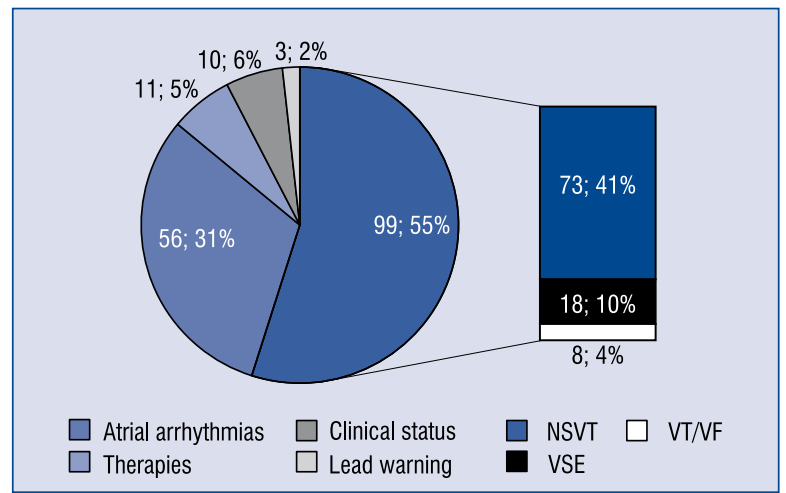

Figure 2. Medtronic CareLink Express ${ }^{\circledR}$ event notifications; NSVT - non-sustained ventricular tachycardia; VSE — ventricular sensing episode; VT/VF — ventricular tachycardia/ventricular fibrillation.

\section{Results}

A total of 119 patients implanted with ICD/ CRT-D (101/18 respectively) were enrolled in the study from April 2013 to December 2013. Mean age of the study population was $64 \pm 14$ years $(63 \pm 14$ and $69 \pm 12$ for ICD and CRT-D group, respectively). The majority of patients were males $(86 \%)$. Mean time interval from device implantation to the assessed follow-up visit was $77 \pm 58$ weeks. Patient characteristics are presented in Table 1.

Data was analyzed from 129 consecutive MCLE transmissions (109 from ICD patients, 20 from CRT-D patients), each RC was followed by a conventional in-person evaluation (SC). All patients managed to perform the transmission owing to a short course and a graphic manual.

179 event notifications were registered in $96(74.4 \%)$ transmissions (Fig. 2). Most reports concerned nonsustained ventricular tachycardia.

Device reprogramming was performed during $100(77.5 \%)$ SCs. 333 changes were made in device settings (Fig. 3). 


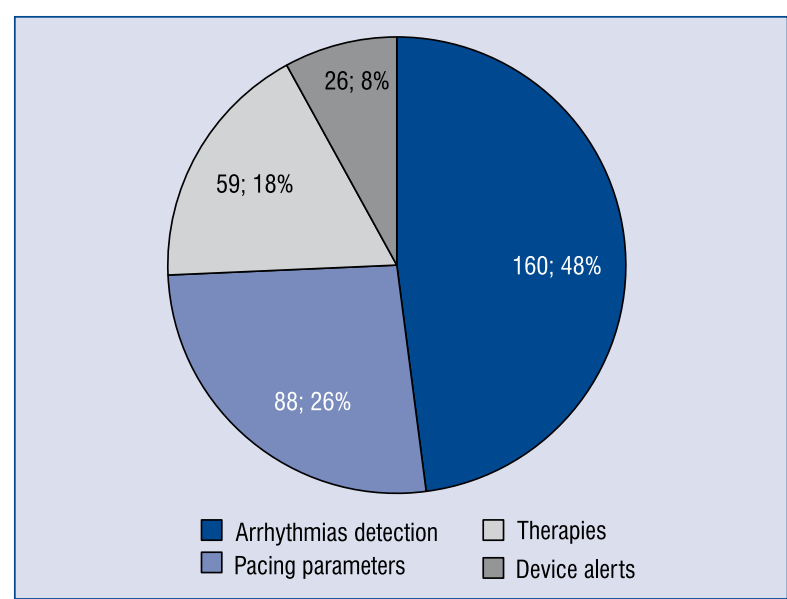

Figure 3. Device setting modifications.

Nevertheless, only 40 modifications (including 27 device programming and 13 medication changes) in 38 SCs were clinically significant and necessary in terms of patient safety. These actions were utilized to assess the efficiency and safety. The conclusions were based on the sensitivity and specificity of the MCLE system.

Sensitivity of MCLE event notifications with regards to detection of a patient requiring clinically significant device reprogramming and/or changes in pharmacological treatment was $86.8 \%$, specificity was $30.8 \%$ (positive and negative predictive value $34.4 \%$ and $84.9 \%$, respectively).

A statistically significant difference between the number of clinically significant interventions among patients with and without MCLE alarms was observed (33 [86.8\%] vs. 5 [13.2\%], $\mathrm{p}=0.037$ ) (Table 2).

\section{Discussion}

The number of implanted electrotherapy devices is constantly rising both in Poland and in Europe [4]. This trend negatively affects physicians who manage patients after electrostimulation procedures creating an increased workload in control centers.
The population with implanted ICD/CRT-D are at high risk of cardiovascular events and ventricular/ supraventricular arrhythmias [7, 8]. An improvement of prognosis and quality of life requires alertness and optimization of therapy. Adequate clinical management is essential for effective prevention of hospitalization, cardiovascular complications and progression of underlying disease.

Remote controls systems may be an answer to the increasing workload in cardiac electrotherapy centers. The benefits of RCs were proven in a series of clinical trials and include: reduction of inadequate interventions of an implanted device, lengthening of its working time and reduced incidence of mortality and hospitalization [9-12]. RC systems can automatically generate alerts reporting arrhythmias or device malfunctioning. This information allows the electrophysiology team to identify patients requiring immediate changes in therapy [13-15].

Nevertheless, SCs remain necessary in the population of patients with implantable electrotherapy devices $[1,2]$. Current guidelines recommend $\mathrm{SC}$ at least once a year in subjects with a high-energy electrotherapy device.

According to available research, this is the first European study to assess MCLE functionality in the optimization of the control process of ICD/CRT-D patients. Results of the trial suggest that MCLE may facilitate follow-up procedures in this group.

It must be stressed that MCLE has successfully enabled the identification of patients requiring clinically significant device reprogramming and changes in pharmacological treatment (sensitivity $86.8 \%$; negative predictive value $84.9 \%$ ). This clearly demonstrates that the use of MCLE system is safe due to its ability to indicate problematic patients.

However, it is not possible to discriminate between patients that do and do not require a SC solely based on the generation of an MCLE alarm. Based on previous research regarding $\mathrm{RC}$ systems it is believed that there are some improvements which could increase the effectiveness of MCLE:

Table 2. Rate of clinically significant changes in device programing or pharmacotherapy among patients with and without Medtronic CareLink Express ${ }^{\circledR}$ (MCLE) event notifications.

\begin{tabular}{lccc}
\hline Endpoint & $\begin{array}{c}\text { Patients with MCLE } \\
\text { event notifications }\end{array}$ & $\begin{array}{c}\text { Patients without MCLE } \\
\text { event notifications }\end{array}$ & P \\
\hline Significant device reprograming & $23(85.2 \%)$ & $4(14.8 \%)$ & 0.149 \\
Medication changes & $1(7.7 \%)$ & $12 ;(92.3 \%)$ & 0.119 \\
Any clinically significant intervention & $33(\mathbf{8 6 . 8 \% )}$ & $\mathbf{5 ( 1 3 . 2 \% )}$ & $\mathbf{0 . 0 3 6}$ \\
\hline
\end{tabular}


- prioritization of automatic alarms - selection of MCLE alerts which were generated in emergency situations and require urgent SC;

- elaboration of risk scales based on selected alarms and clinical parameters (patient clinical characteristics, physical examination);

- creation of self-learning system capable of real-time evaluation of transmissions via MCLE.

The aforementioned features could help analyze indications for SC and generate suggested proceeding based on potential benefits from device reprogramming or pharmacotherapy changes.

Medtronic CareLink Express ${ }^{\circledR}$ enables storage of detailed data about implanted device parameters, a patient's clinical state, and information about the control process.

One cannot overestimate the beneficial influence of MCLE on a patients' self-awareness. Successive reports mention a wide range of difficulties patients have with implanted cardiac electrotherapy devices and experience during $\mathrm{RCs}$ performed with the help of Medtronic CareLink (MCL). In Tele $\mathrm{HF}$ trial, $14 \%$ of subjects belonging to the remote monitoring group did not perform a single transmission during the whole study [14]. An interesting and up-to-date analysis was also published in "Herz" by Siebermair et al. [16], who recorded the frequency of failure in performing a transmission via MCL and investigated the causes that led to the failure. A study group comprised of close to 160 subjects, $24 \%$ did not register a single transmission during a 16-month observation. This situation took place despite at least two notifications sent to them by researchers. It is likely that this observation resulted from patient non-compliance rather than from technical difficulties associated with transmissions. The non-compliance might have been caused by mental overload associated with MCL installation, insufficient engagement and cooperation of the physicians and fear for security of a patient's personal data. Nevertheless, ambulatory interrogations via MCLE and MCL are identical to the ones performed with the help of MCLE before the first SC in the present trial. The process of providing consent for participation in the trial also looks similar. It seems that patients provided with MCLE cooperate much better than patients using other systems, such as MCL.

Finally, it is worth mentioning that all patients showed a positive attitude toward MCLE register. A single, brief training on the station, in most situations carried out in the presence of at least one member of the family, was generally sufficient for independent execution of consecutive inter- rogation. No patient refused to provide informed consent for participation in the MCLE register.

\section{Limitations of the study}

The main limitation of this study is that it presents the experience of a single center. MCLE is available in few cardiology units. According to available research, the presented clinic is the only one to have introduced MCLE in day-to-day monitoring of patients in Poland. SCs of the patients included in the trial were performed by one group of specialists following unified standards of implantable device programming. One investigator was responsible for teaching the patients how to perform a transmission via MCLE during the whole trial.

\section{Conclusions}

Medtronic CareLink Express ${ }^{\oplus}$ is a RC system designed for clinical supervision of patients with implanted cardiac electrotherapy devices. It enables high-sensitivity identification of patients in need of clinically significant device reprogramming or changes in pharmacotherapy during an SC.

Conflict of interest: Marcin Grabowski - speaker honoraria and clinical trials with Medtronic; Andrzej Cacko - speaker honoraria and clinical trials with Medtronic; Marcin Michalak — speaker honoraria and clinical trials with Medtronic; Pawef Balsam - speaker honoraria with Medtronic. Other authors declare no conflict of interest.

\section{References}

1. Dubner S, Auricchio A, Steinberg JS, et al. ISHNE/EHRA expert consensus on remote monitoring of cardiovascular implantable electronic devices (CIEDs). Europace. 2012; 14(2): 278-293, doi: 10.1093/europace/eur303, indexed in Pubmed: 22232544.

2. Wilkoff B, Auricchio A, Brugada J, et al. HRS/EHRA Expert Consensus on the Monitoring of Cardiovascular Implantable Electronic Devices (CIEDs): Description of Techniques, Indications, Personnel, Frequency and Ethical Considerations. Heart Rhythm. 2008; 5(6): 907-925, doi: 10.1016/j.hrthm.2008.04.013.

3. Raatikainen MJ, Arnar DO, Zeppenfeld K, et al. Current trends in the use of cardiac implantable electronic devices and interventional electrophysiological procedures in the European Society of Cardiology member countries: 2015 report from the European Heart Rhythm Association. Europace. 2015; 17 Suppl 4: iv1-i72, doi: 10.1093/europace/euv265, indexed in Pubmed: 26286028.

4. Arribas F, Auricchio A, Wolpert C, et al. The EHRA White Book. Europace. 2012; 14 Suppl 3: iii1-ii55, doi: 10.1093/europace/ eus256, indexed in Pubmed: 22923164.

5. Piotrowicz R, Grabowski M, Balsam P, et al. ["Baltic Declaration"--telemedicine and mHealth as support for clinical processes in cardiology. The opinion of the Committee of Infor- 
matics and Telemedicine of the Polish Society of Cardiology and Telemedicine Clinical Sciences Committee of the PAS]. Kardiol Pol. 2015; 73(7): 575-584, doi: 10.5603/KP.2015.0131, indexed in Pubmed: 26189477.

6. Ahmed I, Patel AS, Balgaard TJ, et al. Technician-Supported Remote Interrogation of CIEDs: Initial Use in US Emergency Departments and Perioperative Areas. Pacing Clin Electrophysiol. 2016; 39(3): 275-281, doi: 10.1111/pace.12798, indexed in Pubmed: 26647906.

7. Ponikowski P, Voors A, Anker S, et al. 016 ESC Guidelines for the diagnosis and treatment of acute and chronic heart failure: The Task Force for the diagnosis and treatment of acute and chronic heart failure of the European Society of Cardiology (ESC). Developed with the special contribution of the Heart Failure Association (HFA) of the ESC. Eur Heart J. 2016; 37(27): 2129-2200, doi: 10.1093/eurheartj/ehw128.

8. Priori SG, Blomström-Lundqvist C, Mazzanti A, et al. 2015 ESC Guidelines for the management of patients with ventricular arrhythmias and the prevention of sudden cardiac death: The Task Force for the Management of Patients with Ventricular Arrhythmias and the Prevention of Sudden Cardiac Death of the European Society of Cardiology (ESC). Endorsed by: Association for European Paediatric and Congenital Cardiology (AEPC). Eur Heart J. 2015; 36(41): 2793-2867.

9. Mabo P, Victor F, Bazin P, et al. A randomized trial of long-term remote monitoring of pacemaker recipients (the COMPAS trial). Eur Heart J. 2012; 33(9): 1105-1111, doi: 10.1093/eurheartj/ ehr419, indexed in Pubmed: 22127418.

10. Varma N, Epstein AE, Irimpen A, et al. Efficacy and Safety of Automatic Remote Monitoring for Implantable Cardioverter-Defibrillator Follow-Up: The Lumos-T Safely Reduces Routine Of- fice Device Follow-Up (TRUST) Trial. Circulation. 2010; 122(4): 325-332, doi: 10.1161/circulationaha.110.937409.

11. Guédon-Moreau L, Lacroix D, Sadoul N, et al. ECOST trial Investigators. A randomized study of remote follow-up of implantable cardioverter defibrillators: safety and efficacy report of the ECOST trial. Eur Heart J. 2013; 34(8): 605-614, doi: 10.1093/ eurheartj/ehs425, indexed in Pubmed: 23242192.

12. Crossley G, Boyle A, Vitense H, et al. The CONNECT (Clinical Evaluation of Remote Notification to Reduce Time to Clinical Decision) Trial. J Am Coll Cardiol. 2011; 57(10): 1181-1189, doi: 10.1016/j.jacc.2010.12.012.

13. Slotwiner D, Varma N, Akar JG, et al. HRS Expert Consensus Statement on remote interrogation and monitoring for cardiovascular implantable electronic devices. Heart Rhythm. 2015; 12(7): e69-100, doi: 10.1016/j.hrthm.2015.05.008, indexed in Pubmed: 25981148.

14. Kotb A, Cameron C, Hsieh S, et al. Comparative effectiveness of different forms of telemedicine for individuals with heart failure (HF): a systematic review and network meta-analysis. PLoS One. 2015; 10(2): e0118681, doi: 10.1371/journal.pone.0118681, indexed in Pubmed: 25714962.

15. Lunati M, Gasparini M, Santini M, et al. Follow-up of CRTICD: implications for the use of remote follow-up systems. Data from the InSync ICD Italian Registry. Pacing Clin Electrophysiol. 2008; 31(1): 38-46, doi: 10.1111/j.1540-8159.2007.00923.x, indexed in Pubmed: 18181908.

16. Siebermair J, Clauss S, Martens E, et al. Remote monitoring of implantable cardioverter-defibrillators. Problems and implications using a telemonitoring system. Herz. 2015; 40 Suppl 2: 110-118, doi: 10.1007/s00059-014-4080-7, indexed in Pubmed: 24848864 . 